\title{
Interactive comment on "Use of remote-sensing reflectance to constrain a data assimilating marine biogeochemical model of the Great Barrier Reef" by Emlyn M. Jones et al.
}

\author{
D. A. Ford (Referee)
}

david.ford@metoffice.gov.uk

Received and published: 6 June 2016

This study assimilates ocean colour data into a coastal biogeochemical model, and aims to show that using remote-sensing reflectance in the assimilation provides better results than using chlorophyll. This is done by applying the OC3M algorithm to both observed and modelled remote-sensing reflectance, rather than simply using the chlorophyll concentration from the surface level of the model. The study concludes that the former approach gives better results than the latter, and that the resulting assimilation improves model results compared with independent in situ observations, and gives skilful forecasts.

Discussion paper

The paper provides useful and novel results, but I'm not convinced that the authors 
have achieved what they claim to have done. The paper claims to have assimilated remote-sensing reflectance, and shown this to be superior to the assimilation of chlorophyll. However, unless I've misunderstood, what's actually been assimilated is still chlorophyll, just with a more intelligent construction of the observation operator than has been used in other schemes. Whilst still novel, important, and of interest to the BG community, the work does not seem to be quite as ground-breaking as the paper claims.

It might be that l'm not doing the paper justice, but in that case the methods need to be much more clearly explained.

I will go through the paper providing my comments, before concluding with my recommendations. I'll note here that the manuscript could have done with proofreading before submission. There are typos throughout, some of the explanations are unclear, and figure, table and section references are often missing or incorrect. Rather than going through all of them in this review, I'll instead recommend that the authors give the manuscript a thorough proofreading before resubmission.

\section{Specific comments:}

Section 1 - The introduction would benefit from a clearer description of the ocean colour processing chain, where different products (e.g. $\mathrm{R}_{r s}, \mathrm{~K}_{d}$, Chl-a, etc) fit into that, and what their errors are.

The introduction should also include a brief discussion of previous ocean colour assimilation studies, especially those that have assimilated products other than chlorophyll, such as Shulman et al. (2013) and Ciavatta et al. (2014).

Section 2.2 - state here that you convert to OC3M yourself, even if the details are still given in a later section. What's the time resolution of the data?

Section 2.3 - a lot of information is given in the appendix, but you don't seem to refer to it anywhere.

Printer-friendly version

Discussion paper 
Section 2.4 - state explicitly that $a$ is absorption and $b_{b}$ is backscattering, and what $z_{0}$ and $z_{1}$ represent.

Section 2.5.1 - please ensure that notation, italicisation, etc. is consistent, describe the localisation operator, comment on whether 36 ensemble members is sufficient, and whether log-transformation is sufficient for normalisation.

Please also clarify what time window you use - 5-day window, 1-day window every 5 days, are 5-day composites or daily data assimilated?

Model formulation, model physics, fluxes, etc. also introduce sources of error.

Section 2.5.2 - I can see why the term's been used, but "super-observations" typically refer to spatially aggregated observations, rather than a variable transformation, so this is potentially confusing.

By applying the OC3M algorithm to the observed remote-sensing reflectance, you're essentially just creating a standard ocean colour chlorophyll product, is this right? I don't understand how this is any different to just assimilating a standard off-the-shelf OC3M chlorophyll product from (e.g.) NASA, which is what you're trying to get away from?

Furthermore, whilst this has been discussed, l'm not convinced about the use of OC3M rather than a regional algorithm that's more appropriate for the region. This should either be justified more strongly, or a regional algorithm compared as well.

Both here and later on, the discussion of what is and isn't linear, and the extent to which this matters, needs to be clearer.

You state that using super-observations "eliminates the possibility of cross-correlation". It will do so between the bands, but there will still be cross-correlations due to nearby observations having similar error characteristics.

The methodology of the different experiments should be explained in this section rather

Printer-friendly version

Discussion paper
Interactive

comment 
than in the results section. Please include the dates that have been run for.

Either here or elsewhere in the paper, please discuss more fully how your approach compares with the typical NWP approach of using a radiative transfer model to convert the model variables, and assimilating the radiances directly.

Section 3.1 - please provide a reason why 14/7/2013 was chosen for Fig. 2 - I assume this date is representative?

Section 3.2 - define "adaptive ODE integrator".

Whilst definitely an indication, a line plot of only nine cycles' worth of mean increments isn't really enough to convince me that using model OC3M rather than model surface level chlorophyll is giving more realistic model chlorophyll. I would like to see some comparison against in situ chlorophyll observations for all the model runs.

I would also like to see some more discussion, either here or later in the paper, about why EXP1 performed so poorly it could only be run for nine assimilation cycles. This is the standard procedure that other groups use successfully, so for the community to be convinced that using an alternative method will be beneficial generally, and it's not just due to how your system is set up, some more information would be helpful.

Section 3.3 - whilst observations from $t+5$ days haven't been assimilated, they will share a lot of characteristics with observations which have, since they come from the same source. I would therefore only class them as "semi-independent", rather than fully independent.

If OC3M follows a logarithmic distribution, would it be more appropriate to calculate statistics on log-transformed OC3M? Especially since you state that the distribution of errors is positively skewed.

Printer-friendly version

Be clear, both here and in the caption for Fig. 4, that your depth ranges refer to the bottom depth of the water column, rather than intervals within the water column.

Discussion paper 
Section 3.3.1 - you pick a single cycle (the final one?) and make clear that the features you describe are just for "this particular analysis cycle". This is fine, but please make some mention of how representative it is of other cycles.

The overlaid observations are too small to be seen without zooming in to about $400 \%$ on the pdf - on a printed copy they're just black blobs. Please make sure these can be seen in the final version.

Perhaps because they're on different pages, or because the colour scale is saturated, but Fig. 5a and 7a look very similar to me.

Section 4 - whilst your results have "been achieved by explicitly assimilating like-forlike variables", you haven't actually shown any assimilation results against observations from not doing so.

You state that "Our approach of simulating the observation is the opposite to the conversion of observed remote-sensing reflectances into modelled variables, e.g. the assimilation of ... Chl-a." But as far as I can tell, this is exactly what you've done you've used the OC3M algorithm to convert the remote-sensing reflectance to Chl-a. Please tell me if l've misunderstood! It's true that you've processed the model and observations in the same way, which is a good thing to have done and novel in biogeochemical assimilation, but it's not the same as directly assimilating the remote-sensing reflectance.

The final paragraph of this section needs re-writing to be clearer (currently it advocates "remote-sensed reflectance" as an alternative approach to "remote-sensing reflectances"), and to place it in the context of differing model complexities and regions of interest.

Section 5 - you state you "halve the forecast error compared to simply assuming the OC3M is directly related to the model prediction of surface total Chl-a" - has this been shown? Is this just based on Fig. 3, or something else? 
If non-ocean colour-specific sensors can be reliably assimilated then that would indeed be a great breakthrough. But given that you still appear to transform the remotesensing reflectance to chlorophyll, will the current approach still require the use of ocean colour-specific sensors? Please explain this more clearly.

Finally, please state the variables and units in your Table descriptions.

\section{Recommendations:}

Assuming that I haven't misunderstood what's already been done, then ideally I would like to see the direct assimilation of the remote-sensing reflectance, as that would make for a very important step forward in biogeochemical assimilation. However, I acknowledge that this would involve a considerable amount of extra effort, and would in effect constitute a separate study.

Therefore, I'm happy that the current work is suitable for publication in BG, provided that the authors are clear about what has been done (the like-for-like processing of model and observations, rather than the direct assimilation of remote-sensing reflectance), the comments above are addressed, and the manuscript is tidied up. In particular, I would like to see greater comparison between the different experiments, with all runs compared to in situ observations. Furthermore, either a regional algorithm should be included in the comparison, or a much stronger justification should be provided for why this hasn't been done. Whilst in this case the direct assimilation of remote-sensing reflectance doesn't seem to have been performed, it is an important step towards it.

\section{References:}

Ciavatta, S., Torres, R., Martinez-Vicente, V., Smyth, T., Dall'Olmo, G., Polimene, L., Allen, J. I. (2014). Assimilation of remotely-sensed optical properties to improve marine biogeochemistry modelling. Progress in Oceanography, 127, 74-95.

Shulman, I., Frolov, S., Anderson, S., Penta, B., Gould, R., Sakalaukus, P., Ladner, S. (2013). Impact of bio-optical data assimilation on short-term coupled physical, bio-

Printer-friendly version

Discussion paper 
optical model predictions. Journal of Geophysical Research: Oceans, 118(4), 22152230.

Interactive comment on Biogeosciences Discuss., doi:10.5194/bg-2016-168, 2016. 